\title{
TRADUCCIÓN ECONÓMICA AL GALLEGO: PAUTAS DE ACTUACIÓN ANTE LA PRESENCIA DE PRÉSTAMOS Y CALCOS EN EL LÉXICO ECONÓMICO
}

\author{
Marta García González \\ mgarciag@uvigo.es \\ Universidade de Vigo
}

\section{Resumen}

En línea con trabajos de temática similar (Russo 2002; Orts \& Almela 2009; Orts \& Almela 2012), el presente estudio analiza los mecanismos de creación de neologismos del campo de la economía en la lengua gallega, prestando especial atención a la entrada de extranjerismos, calcos semánticos, préstamos adaptados y castellanismos propiciada por la actual situación de crisis mundial. De esta forma, se pretende proporcionar pautas de actuación en el uso de estos elementos léxicos a los estudiantes de traducción y futuros traductores al gallego de textos económicos, ante la carencia de recursos de referencia y de la experiencia necesaria para tomar decisiones en esta materia. Se trabaja para ello con dos corpus textuales ad hoc compilados de manera expresa para el estudio. El primero de ellos es un corpus monolingüe en lengua gallega conformado por artículos periodísticos de temática económica publicados en diarios de la comunidad autónoma y por artículos de investigación extraídos de la Revista Galega de Economía, que se edita semestralmente en lengua gallega y castellana. El segundo es un corpus bilingüe comparable conformado por artículos de la citada revista en sus versiones gallega y castellana. El análisis de los corpus apunta a la existencia de patrones de uso diferentes de los extranjerismos, préstamos adaptados y calcos en los textos económicos especializados y en los de divulgación, así como hacia una coincidencia en los patrones de uso de estos elementos en la lengua gallega y española. 


\begin{abstract}
"Financial Translation into the Galician Language: Guidelines for Decision-Making in the Use of Loanwords and Calques in the Financial Discourse"

In line with previous studies (Russo 2002; Orts \& Almela 2009; Orts \& Almela 2012), the present paper analyses the mechanisms that the Galician language has for neologism creation in financial documents, particularly focusing on the use of loanwords and calques. Two ad hoc text-based corpora were compiled for the study: (i) a Galician monolingual corpus made up of Galician press articles and Galician research articles drawn from the bilingual journal Revista Galega de Economia; and (ii) a bilingual corpus comprised of the Galician and Spanish versions of the articles from the aforementioned journal. Results point to the existence of different usage patterns of loanwords and calques in specialized economic texts and in economic news-items, and of similar usage patterns of loanwords and calques in research articles in Galician and Spanish.
\end{abstract}

Palabras clave: Discurso especializado económico. Extracción terminológica. Gallego. Lengua minorizada. Neologismo.

Keywords: Economic specialized discourse. Galician language. Minoritized language. Neologism. Terminological extraction.

Manuscript received on June 30, 2015 and accepted for publication on October 27, 2015. 


\section{Introducción}

El lenguaje económico, al igual que otros lenguajes de especialidad, se nutre en gran medida de términos procedentes de la lengua inglesa, por ser esta la lengua franca de comunicación entre operadores de diferentes países (Furiassi, Pulcini \& Rodríguez González 2012: 3). La forma en que estos términos se incorporan al discurso de cada lengua depende, entre otros factores, de la capacidad que dicha lengua tiene para desarrollar neologismos a la misma velocidad en que los términos se incorporan al discurso especializado, así como de los procedimientos lingüísticos empleados con mayor frecuencia para este desarrollo. Sin embargo, a diferencia de lo que puede suceder con otros lenguajes, como el científico o el jurídico, cuyo uso se encuentra más limitado a sus ámbitos de especialización, el lenguaje económico tiene una mayor presencia en contextos semiespecializados o divulgativos, principalmente en los medios de comunicación, tanto orales como escritos. Esta presencia, en un entorno en el que la inmediatez de la noticia prima en muchas ocasiones por encima de la calidad de los contenidos, pero también de la forma, provoca la entrada en el lenguaje económico de términos a veces desafortunados como es el caso de los fondos carroñeros o fondos buitre, las hipotecas subprime o las más recientes hipotecas underwater o hipotecas bajo el agua.

En el contexto de las lenguas minorizadas, cuyo uso en determinados ámbitos no se encuentra todavía normalizado, la entrada de nuevos términos en la lengua se ve influida, además, por la forma en que dicha entrada se ha producido en la lengua dominante con la que convive. Mientras los discursos no especializados discurren muchas veces en paralelo, los especializados con frecuencia son elaborados por las mismas personas en ambas lenguas. Esta situación resulta especialmente problemática para la docencia de la asignatura de traducción económica en la combinación inglés-gallego, por implicar una falta de fuentes de referencia fiables para un colectivo que carece de la subcompetencia necesaria para tomar decisiones informadas. Esto se agrava por la inexistencia de pautas de actuación homogéneas que indiquen a los futuros traductores cómo deben actuar ante un problema terminológico de esta naturaleza. 
Son numerosos los trabajos que en los últimos años se han centrado en el estudio de la influencia del inglés como fuente de incorporación de términos y expresiones en diferentes lenguas europeas (Crystal 2002; Fiedler 2011; Martí Solano 2012; Oncíns Martínez 2009, 2012, 2014; Rodríguez González 2004; Rozumko 2012). Su estudio en el caso de las lenguas minorizadas, sin embargo, resulta menos frecuente. Siguiendo la línea de trabajos previos de temática similar (Russo 2002; Orts \& Almela 2009; Orts \& Almela 2012), en las páginas siguientes analizamos los mecanismos de creación de neologismos del campo de la economía en la lengua gallega, prestando especial atención a la entrada de extranjerismos, calcos semánticos, préstamos adaptados y castellanismos propiciada por la actual situación de crisis mundial. Desde una perspectiva didáctica, a partir del uso de metodologías sencillas de explotación de corpus, a semejanza de las presentadas en Zanettin, Bernardini y Stewart (2003), buscamos definir un modelo de búsqueda sistemática de términos económicos para el aula de traducción económica al gallego y, en la medida de lo posible, establecer patrones de actuación para la selección entre un extranjerismo y el término equivalente en lengua gallega en función de las expectativas de los usuarios finales de los textos traducidos (expertos /no expertos).

Para ello, realizamos una breve descripción de la presencia de la lengua gallega en distintos ámbitos en los que se generan discursos económicos o comerciales y revisamos los principales problemas del léxico económico gallego y los mecanismos más habituales de dicha lengua para la creación de neologismos. A continuación, trabajamos con dos corpus textuales ad hoc compilados de manera expresa para el estudio: el primero de ellos es un corpus monolingüe en lengua gallega conformado por artículos periodísticos de temática económica publicados en diarios de la comunidad autónoma y por artículos de investigación extraídos de la Revista Galega de Economía, que se edita semestralmente en lengua gallega y castellana. Por su parte, el segundo constituye un corpus bilingüe comparable conformado por artículos de la citada revista en sus versiones gallega y castellana. El primer corpus se utilizará para determinar la presencia de extranjerismos, calcos semánticos, préstamos adaptados y castellanismos en los textos compilados y para establecer si existen diferencias en estos usos en función del tipo de texto (especializado frente a informativo) y del autor (experto/semiexperto). A partir del segundo corpus se analizará si los anglicismos, préstamos, calcos y castellanismos identificados en los artículos de la revista de investigación coinciden con los empleados en la versión castellana de los mismos artículos, con el fin 
de determinar si existe una correlación entre los usos de estos términos en una y otra lengua o si los autores recurren a mecanismos diferentes.

\section{El uso de la lengua gallega en la economía}

\subsection{Presencia de la lengua en los sectores público y privado}

De acuerdo con el informe sectorial del Observatorio da Lingua Galega (OLG), publicado en 2007 (EOSA Consultores 2007: 33), la presencia de la lengua gallega en el ámbito de la economía es escasa si se la compara con la del castellano. Concretamente, en el ámbito de la pequeña empresa, el porcentaje de uso del gallego se sitúa por debajo del $45 \%$ en las comunicaciones orales y del 20\% en las escritas, cifras que se repiten en los ámbitos del comercio y de la banca. Por lo que se refiere a su uso en sitios web empresariales, el estudio reveló que en torno a un $83 \%$ de las empresas tenían su sitio solo en castellano, frente a un $11,5 \%$ que la tenían tanto en castellano como en gallego y únicamente un 3,8\% la tenían solo en gallego (EOSA Consultores 2007: 35). Estas cifras se reducen aún más cuando se analiza la información social y financiera que las empresas publican en sus sitios, de acuerdo con García González (2013) donde, sobre una muestra de 2.000 empresas gallegas, solo se localizó una que incluyese en su página documentación social en gallego.

En la administración pública, la lengua gallega se utiliza de manera generalizada en los procedimientos que emanan de las diferentes consellerías del gobierno autonómico, por lo que resulta posible disponer de abundante información redactada en esa lengua sobre contratación pública, presupuestos autonómicos, planes estratégicos, política financiera, tributación, inversión pública y apoyo a la inversión privada, etc. En las diputaciones y en los ayuntamientos, la presencia del gallego es más variable, aunque en general también es posible encontrar en lengua gallega toda la información relacionada con la gestión económica, así como todos los formularios necesarios para la interacción entre los usuarios y la administración. En la administración estatal y las subdelegaciones del gobierno en Galicia el acceso a información en lengua gallega es mucho más limitada. Las propias páginas web de los ministerios de Economía y Competitividad y de Hacienda y Administraciones Públicas, pese a disponer en ambos casos de la opción lingüística 'gallego', carecen de documentación traducida al gallego de temática macro o microeconómica.

Por lo que se refiere a la presencia del gallego en la prensa escrita, en la actualidad, tras el cierre de anosaterra.org y Vieiros, la oferta de periódicos en lengua gallega es escasa y se limita principalmente al medio digital: Galiza Hoxe, Praza Pública, Sermos Galiza, Galicia Confidencial, Diário Liberdade y 
Galiciaé son las publicaciones de mayor relevancia. A excepción de Galiza Hoxe, que comenzó a publicarse en $2003^{1}$ vinculada a El Correo Gallego, y Galiciaé, creada en 2007 y perteneciente al grupo El Progreso, en todos los demás casos se trata de publicaciones digitales creadas en los últimos años (Galicia Confidencial y Diário Liberdade en 2009, Praza Pública y Sermos Galiza en 2012,) por iniciativa de periodistas independientes y de la sociedad civil, sin vinculación con las empresas periodísticas del sector. En ellas se publican todo tipo de noticias y disponen de una sección específica dedicada a la economía. El uso del gallego en el resto de los periódicos de ámbito regional se sitúa en torno a un $6,5 \%$ mientras que su presencia es anecdótica o inexistente en los de ámbito estatal (EOSA Consultores 2008a: 24).

En el entorno académico, aunque el gallego es la lengua oficial de las tres universidades gallegas y por lo tanto la utilizada por defecto en toda la documentación administrativa y académica que emana de ellas, así como en la interacción oral con los usuarios, la presencia de la lengua en las aulas se sitúa en torno al 24\% según datos del OLG (EOSA Consultores 2008b: 109). En la investigación, la presencia es aún menor. Nogueira (2013: 26), en un estudio encargado por la Comisión Interuniversitaria de Política Lingüística, cifra el uso exclusivo del gallego como lengua de investigación en un 8\%, a lo que se suma un $36 \%$ de los investigadores que afirman combinar castellano y gallego en su actividad. En el ámbito de las ciencias sociales, estos valores se sitúan en el $9 \%$ y el $56 \%$, respectivamente. En materia económica, existe al menos una revista que se edita íntegramente en versión bilingüe español/ gallego, Revista Galega de Economía, editada por la Universidade de Santiago de Compostela desde 1992.

\subsection{El léxico económico gallego}

Giménez \& Lores (2005: 7-8) identifican cuatro problemas básicos para la elaboración de un vocabulario léxico científico en el ámbito de la economía:

- La influencia del español y, sobre todo en los últimos años, también del inglés, que es muy fuerte sobre todo en el caso de los términos de nueva creación. El recurso al portugués como fuente alternativa, por otro lado, se ve muchas veces frustrado por la tendencia cada vez mayor de los economistas portugueses a importar directamente los nuevos términos de la literatura económica en inglés en lugar de traducirlos a su lengua.

1. Si bien su edición impresa dejó de publicarse en 2011. 
- El hecho de que la misma terminología científica económica posea diferentes usos y grafías en distintas lenguas. Estas variaciones están presentes en las dos lenguas que más influencia ejercen sobre la entrada de terminología en la lengua gallega, esto es, el inglés y el español. Así, por ejemplo, encontramos variaciones en la lengua inglesa tanto en el nivel gráfico (labor/labour) como terminológico (inventory/stock), mientras que en español las variaciones son principalmente terminológicas (equilibrar/balancear, renta/ingreso, tasa/ tipo). Algunas de estas variaciones se han incorporado ya a la lengua gallega (renda/ingreso, taxa/tipo), donde conviven con otras propiciadas por la combinación de préstamos con términos autóctonos (gasto/ despesa, orzamento/presuposto, aforro/poupanza, demanda/procura, devengar/devindicar).

- A raíz del problema anterior y a causa de la inexistencia de una norma o criterio de adopción de terminología económica, el esfuerzo por normalizar el estatus de la lengua a fuerza de aumentar su presencia en todos los ámbitos antes de desarrollar su corpus ha provocado la adopción de soluciones poco acordes con la pureza léxica.

- Las guerras ortográficas que durante muchos años marcaron el desarrollo de la lengua gallega y que provocaron un rechazo de la utilización de soluciones terminológicas acordes con la lengua portuguesa, al tiempo que afectaron "o noso desenvolvemento cotián como usuarios do galego científico, maiormente nas aulas docentes e, nalgúns casos, na comunicación científica e cara á sociedade" (Giménez \& Lores 2005: 8)

El resultado es un léxico no normalizado, muy influido por la lengua española pero también por la inglesa y la portuguesa, en la que conviven diferentes formas para muchos términos entre las que el usuario no experto no siempre sabe cuál debe escoger. Se une a este problema la inexistencia de diccionarios especializados monolingües o bilingües y el hecho de que los recursos terminológicos elaborados a partir de corpus se basan en textos en gallego producidos en este mismo contexto de falta de normalización de la lengua especializada, lo que perpetúa sus carencias.

\subsection{Formas de incorporación de nuevas unidades terminológicas a la lengua gallega}

Partiendo de la definición propuesta por Auger \& Rousseau (1978: 54) de neologismo como una "unité lexicale de formation récente, une acception 
nouvelle d'un terme existant déjà, ou encore, un terme emprunté depuis peu à un système linguistique étranger" y de su tipología general de la neología, clasificamos los neologismos en tres amplias categorías:

- Neologismos formales: aquellos formados por procedimientos de derivación, composición, sintagmación o abreviación (ej. zapaterista, teledocencia, desfinanciar).

- Neologismos semánticos: aquellos creados por medio de la variación del contenido semántico de una determinada unidad ya existente en la lengua. El cambio puede producirse de la lengua general a una lengua de especialidad, entre dos lenguas de especialidad, de una lengua de especialidad a la lengua general, o incluso por ampliación de contenido semántico de un término de una lengua de especialidad determinada (ej. troica, tejido social).

- Neologismos de préstamo: aquellas unidades tomadas de lenguas extranjeras vivas o muertas, bien directamente (extranjerismo) bien mediante algún tipo de adaptación fonética y gráfica para acomodarse a la nueva lengua de destino (préstamo adaptado) (ej. leasing, online, márketing, tableta). Guerrero Ramos (1997), entre otros autores, hace referencia a la distinción establecida por Tappolet (1913: 54-55) entre 'préstamos por necesidad' o 'denotativos', que son aquellos que designan productos y conceptos nacidos en un país extranjero, para los que no existe un equivalente en la lengua receptora, y 'préstamos de lujo' o 'connotativos', causados por un "mimetismo lingüístico, desarrollado por el prestigio ejercido por un cierto tipo de civilización y cultura, o por ignorancia, papanatismo, etc." (Guerrero Ramos 1997: 37). Sin embargo, al menos en nuestro ámbito de estudio, esta distinción resulta en ocasiones de difícil aplicación, dado que la rápida introducción de conceptos nuevos en el ámbito de la economía y su incorporación a la lengua a través de canales diferentes (académico/ especialista/prensa) provoca con frecuencia la coexistencia de préstamos y términos propios cuyo uso se normaliza de manera diferente en unos discursos que en otros. De esta manera, lo que en un determinado contexto puede considerarse un préstamo de lujo, no lo es tan claramente en un contexto diferente.

Por último, a la clasificación anterior debemos añadir el calco semántico, íntimamente relacionado con el préstamo, que consistiría en la traducción directa de una palabra o término de otra lengua, una práctica de incorporación neológica bastante habitual en el ámbito de la traducción económica 
(grey market/mercado gris; bear market/mercado oso; shark/tiburón; vulture fund Ifondo buitre...).

Araya \& Gómez (1999: 135-160) analizan los aspectos lingüísticos de la neología en lengua gallega por medio de un corpus de textos del género periodístico y llegan a la conclusión de que el procedimiento de creación terminológica más rentable es el de la derivación, algo que coincide con lo que sucede en otras lenguas como el catalán (Cabré, Freixa \& Solé 1997: 67). Con todo, la presencia de préstamos se cifra en más de un 19\% de los cuales, casi las tres cuartas partes eran extranjerismos frente a solo un $26 \%$ de préstamos adaptados. Los autores atribuyen este predominio del extranjerismo frente al préstamo adaptado a las características propias de los textos de prensa, redactados con gran premura y no sujetos a revisión exhaustiva. En cuanto a los datos por origen del préstamo, destaca el inglés seguido de lejos por el portugués y por el italiano. No se consideraron en el estudio, sin embargo, los denominados castellanismos, o préstamos del castellano, que los autores califican de interferencias por desconocimiento de las soluciones autóctonas (Araya \& Gómez 1999: 148). Estos términos sí se tendrán en cuenta en nuestro trabajo, por considerarlos de interés para el alumnado de traducción al gallego, que deben aprender a identificarlos y a eliminarlos de sus textos.

\section{Materiales y métodos}

\subsection{Selección de los textos para la compilación de los corpus}

Antes de presentar los corpus empleados para la realización del trabajo, nos parece relevante incluir los criterios que se han tenido en consideración en el momento de seleccionar los textos. Sinclair (1991: 171) define corpus como "a collection of naturally-occurring language text, chosen to characterize a state or variety of a language". Con su definición, el autor trata de destacar el hecho de que se trata de textos reales y que caracterizan una variedad o un estado concreto de una lengua. A ello se añade la necesidad de que la compilación cumpla unos requisitos mínimos de cantidad y calidad (número de palabras y representatividad), codificación simple y documentación (trazabilidad de las fuentes originales). Además, como indica Pearson (1998: 61), los textos deben representar aquello que se conoce o que se cree que existe (factuality).

Basándose en el informe realizado por EAGLES (Expert Advisory Group on Language Engineering Standards) en 1996, dedicado a los corpus textuales y en Torruella \& Llisterri (1999: 45-77), Corpas (2001: 158-159) presenta una clasificación de los corpus atendiendo a cinco criterios: (i) tipos de textos contenidos; (ii) especificidad de los documentos; (iii) cantidad de 
texto de los documentos; (iv) codificación y anotación; (v) documentación complementaria. ${ }^{2}$

\begin{tabular}{|l|l|l|l|l|l|}
\hline CRITERIO & Tipos de textos & Especificidad & $\begin{array}{l}\text { Cantidad de } \\
\text { texto }\end{array}$ & $\begin{array}{l}\text { Codificación y } \\
\text { anotación }\end{array}$ & $\begin{array}{l}\text { Documentación } \\
\text { complementaria }\end{array}$ \\
\hline $\begin{array}{l}\text { TIPOS DE } \\
\text { CORPUS }\end{array}$ & Corpus extenso & Corpus general & $\begin{array}{l}\text { Corpus } \\
\text { textual }\end{array}$ & $\begin{array}{l}\text { Corpus no } \\
\text { anotado }\end{array}$ & $\begin{array}{l}\text { Corpus } \\
\text { documentado }\end{array}$ \\
\cline { 2 - 6 } & $\begin{array}{l}\text { Corpus } \\
\text { equilibrado }\end{array}$ & $\begin{array}{l}\text { Corpus } \\
\text { especializado }\end{array}$ & $\begin{array}{l}\text { Corpus de } \\
\text { referencia }\end{array}$ & Corpus anotado & $\begin{array}{l}\text { Corpus no } \\
\text { documentado }\end{array}$ \\
\cline { 2 - 6 } & $\begin{array}{l}\text { Corpus } \\
\text { piramidal }\end{array}$ & Corpus genérico & Corpus léxico & & \\
\cline { 2 - 6 } & Corpus monitor & Corpus canónico & & & \\
\cline { 2 - 6 } & Corpus paralelo & $\begin{array}{l}\text { Corpus } \\
\text { cronológico }\end{array}$ & & & \\
\cline { 2 - 6 } & $\begin{array}{l}\text { Corpus } \\
\text { comparable }\end{array}$ & $\begin{array}{l}\text { Corpus } \\
\text { diacrónico }\end{array}$ & & & \\
\hline
\end{tabular}

Figura 1: Clasificación de los corpus lingüísticos. Elaboración propia a partir de Corpas (2001: 157-159).

En lo que concierne al diseño del corpus, Kennedy (1998: 60) afirma que en el diseño y compilación resulta primordial tener en cuenta la validez y la fiabilidad de la investigación realizada a partir del mismo, incluido si el corpus sirve para el objetivo para el que fue concebido. Así, propone tres variables principales que se deberán tener en cuenta:

- Representatividad: la medida en que los textos compilados son representativos de una determinada lengua o género. Como ya indicara Baker (1995: 239), se trata de un aspecto difícil de determinar y en el que entran en juego cuestiones tan subjetivas como el grado de influencia de un texto o su mérito literario. Aunque existen indicadores más objetivos como el número de ejemplares distribuidos de un determinado texto, no siempre es posible determinar el número de sujetos que tuvieron acceso a cada uno de ellos.

- Nivel de permanencia, esto es, si se trata de un corpus estático o dinámico. Como Hunston (2002: 25) afirma, se trata de un aspecto

2. Para una tipología más detallada de los corpus, basada en doce criterios de clasificación (canal de producción, cantidad de palabras, distribución textual, contenido, tamaño de las muestras, codificación/anotación, documentación, período de tiempo, capacidad de actualización, finalidad, selección de las muestras e idiomas), véase Vargas Sierra (2006). 
relacionado con la representatividad en aquellos casos en los que ser representativo implica estar al día con los últimos avances del lenguaje, como sucede en la incorporación terminológica.

- Tamaño: Kennedy (1998: 66) afirma que un corpus grande no tiene por qué ser necesariamente más útil que uno pequeño, en especial si lo que se desea medir es la frecuencia de uso de determinadas palabras. También Bowker \& Pearson (2002: 45) consideran que el tamaño del corpus es un factor subordinado a otras variables, como las necesidades del proyecto, la disponibilidad de datos y el tiempo disponible. Hunston (2002: 26) añade que el tamaño del corpus dependerá también de la disponibilidad de textos adecuados para su procesamiento y análisis, así como de otros factores como la necesidad de anotación manual. Por otra parte, tan relevante o más que el tamaño es el número de textos, sus autores y si se trata de textos completos o de fragmentos seleccionados. Para los corpus ad hoc destinados al estudio de las lenguas de especialidad, Bowker \& Pearson (2002: 49) recomiendan compilar un número elevado de textos completos y dentro de lo posible redactados por diferentes autores, en lugar de optar por escoger un número reducido de fragmentos o textos de un mismo autor.

Respecto del idioma de los textos compilados, Zanettin (2011: 17) considera que la práctica habitual de excluir textos traducidos de los corpus monolingües parece derivar de la idea de que las traducciones no representan una lengua, sino que la distorsionan. En su opinión, sin embargo, la traducción es una actividad legítima de producción textual y los textos traducidos forman una parte de la oferta a la que se enfrentan diariamente los hablantes de una lengua. En el caso de la lengua gallega, en la que la traducción encubierta (House 1981: 188-211) es una práctica habitual (García González 2003: 63), la compilación de un corpus puro de textos originales resultaría a la vez imposible y carente de representatividad. En lo que respecta a los corpus paralelos compilados específicamente para el estudio de la traducción, bien como proceso, bien como producto, Zanettin (2000: 105) indica que existen condicionantes específicos derivados del trabajo con textos originales y textos traducidos. La búsqueda de la representatividad, por ejemplo, puede convertirse en un problema en la compilación de corpus paralelos bidireccionales, dado que los flujos de traducción entre dos pares de lenguas nunca son iguales: no se traducen el mismo tipo de textos del inglés al español que del español al inglés, por ejemplo, como tampoco se traduce lo mismo del español al 
gallego que del gallego al español. Si se intentase compilar una muestra de textos representativos de las traducciones del gallego al español y del español al gallego, lo más probable es que el corpus resultante no fuese comparable.

Teniendo en cuenta los criterios descritos, para el desarrollo de nuestro estudio hemos compilado dos corpus ad hoc, que Pearson (1998: 48) define como "a special purpose corpus, whose composition is determined by the precise purpose for which it is to be used". El primero de ellos es un corpus monolingüe especializado, compuesto por textos informativos de temática económica extraídos de prensa autonómica en lengua gallega y por artículos de investigación. Se trata de un corpus textual, no anotado pero sí documentado, de modo que permita identificar el origen de cada término extraído. Está compuesto por 378.655 palabras extraídas de dos tipos principales de fuentes: la revista especializada Revista Galega de Economía (249.338 palabras repartidas en 29 artículos) y los periódicos Galicia Hoxe (44.448 procedentes de 195 noticias), Vieiros (38.304 procedentes de 114 noticias), Praza Pública (39.856 procedentes de 124 noticias) y Sermos Galiza (41.179 procedentes de 178 noticias). ${ }^{3}$ Pese a no tratarse de un corpus extenso, consideramos que resulta adecuado para el objeto de análisis y que es representativo del tipo de textos de temática económica de mayor consumo. El segundo es un corpus "ad hoc" textual, paralelo, especializado y documento. Se compone de los mismos 29 artículos extraídos de la Revista Galega de Economía para el primer corpus y de las correspondientes versiones en castellano de los mismos (249.513 palabras en gallego y (279.262) en castellano.

Para la extracción de los artículos de prensa se seleccionaron artículos de las secciones de economía de cada una de las publicaciones relacionadas con la crisis económica, con la Unión Europea y con la situación económica y laboral de Galicia. Los textos extraídos de Galicia Hoxe y Vieiros comprenden noticias publicadas entre 2000 y 2011, mientras que los de Praza Pública y Sermos Galiza comprenden noticias de entre 2012 (año en que comenzaron a publicar) y 2014. Dentro de la revista, los artículos se escogieron entre los años 2000 y 2014. Al igual que en el caso de las noticias, se seleccionaron todos aquellos artículos que trataban algún aspecto de la crisis económica, todos los que trataban aspectos relativos a la Unión Europea y los que se centraban en la situación económica y laboral de Galicia.

En lo que se refiere a la lengua original de los textos, de manera general los textos de Vieiros, Praza Pública y Sermos Galiza están redactados

3. La aplicación de una stop list de palabras con significado gramatical dio como resultado una cifra definitiva de 212.998 palabras para la extracción de términos. 
originalmente en gallego, mientras que los de Galicia Hoxe son en su mayoría bien adaptaciones bien traducciones parciales o totales de las noticias del diario autonómico en lengua castellana El Correo Gallego. En el caso de los artículos de la Revista Galega de Economía, en la publicación se recogen tanto textos redactados originalmente en gallego y luego traducidos al castellano, como textos redactados originalmente en castellano y traducidos después al gallego. De acuerdo con las normas de presentación de artículos, en el caso de trabajos originales en lengua gallega, los autores deben enviar también la versión en castellano y la revista se encarga de la revisión lingüística de ambas versiones, mientras que en el caso de los trabajos originales en lengua castellana, la revista se encarga de la traducción al gallego y de la revisión lingüística del castellano. Pese a las consultas realizadas a los editores de la publicación, no ha sido posible obtener una relación exacta de cuáles de los artículos se corresponden con cada una de las dos pautas, si bien las indicaciones apuntaban a que son más frecuentes los casos en los que el original es el castellano y el gallego la traducción. Con todo, consideramos que esta circunstancia no impide el uso del corpus para la investigación presentada, dado que permitirá determinar si existen correlaciones en el uso de los términos en una y otra lengua. En este sentido, de acuerdo con Baker (2004: 172), entre las características diferenciales que la teoría de la traducción ha atribuido tradicionalmente al texto traducido frente al original, se incluyen la tendencia hacia un uso más conservador del lenguaje, la preferencia por formas estándar frente a variantes y regionalismos, el empleo de una variedad ligeramente más formal y la producción de textos más "uniformes" evitando, por ejemplo, la alteración de las secuencias temporales. Cabe comprobar si estas características diferenciales se identifican entre pares de lenguas tan próximas estructuralmente como son el castellano y el gallego, en especial en lo que atañe al uso de extranjerismos, préstamos adaptados y calcos semánticos. De detectarse diferencias en el tratamiento de los términos en unos artículos y no en otros, esta circunstancia podría ser indicativa de la existencia de comportamientos divergentes entre autores y traductores, lo que podría justificar una ampliación del corpus y del estudio. Debe tenerse en cuenta, por otra parte, que la presencia de autotraducción puede contrarrestar esta tendencia, en tanto que el autor actúa ante su propio texto de manera diferente a como lo haría un traductor. 


\subsection{Explotación de los corpus}

Para la localización de los extranjerismos, préstamos adaptados, calcos semánticos y castellanismos en el primer corpus se han aplicado dos metodologías diferentes. En primer lugar, se ha realizado una búsqueda de una selección inicial de extranjerismos, préstamos y calcos del inglés, para la cual se ha partido de la selección inicial realizada por Orts \& Almela (2009) en un trabajo de corte similar realizado para la lengua española, que se completó con el Glosario Financiero de la Crisis de la BBC, el Diccionario Básico de la Crisis de Expansión y el Diccionario de términos económicos y financieros de La Caixa. La misma metodología se ha utilizado para la identificación de castellanismos, recurriendo en este caso a la obra Lingua galega: dúbidas lingüísticas, del ANL de la Universidade de Vigo, que ofrece un listado de los castellanismos de uso más frecuente en la lengua gallega. Ambas búsquedas se han realizado utilizando el programa de concordancias gratuito AntConc 3.2.4., cuya herramienta Concordance nos permitió buscar cada uno de los términos de la selección inicial en todos los textos compilados, contabilizar el número de resultados, consultar el contexto de uso del término y determinar el texto del corpus al que pertenecía. Tras la búsqueda en el corpus de todos los términos del listado inicial, utilizando la herramienta Wordlist del programa de concordancias, generamos un listado de todas las palabras clasificadas por orden de incidencia, para de esta forma comprobar la presencia de otros extranjerismos, préstamos, calcos semánticos y castellanismos que no hubiésemos incluido en nuestros listados iniciales.

Por último, y como complemento a la búsqueda de los términos en los textos compilados, se procedió a comprobar la presencia de cada uno de ellos en una serie de corpus y recursos lexicográficos de la lengua gallega, con objeto de determinar su grado de normalización en la lengua:

- CORGA (Corpus de referencia do galego actual) (1975-2014) Área economía (CORGA).

- Vocabulario galego de economía (2005) (VGE).

- Novas palabras galegas do OLG da Universidade de Vigo (2005) (NPG).

- CLUVI: corpus lingüístico paralelo da Universidade de Vigo (CLUVI) (hasta 2006). ${ }^{4}$

- CTG: corpus técnico do galego (hasta 2006) (CTG).

4. Dentro del CLUVI, se buscó únicamente en el corpus EGAL, de economía gallego-español. 
En el caso del segundo corpus, lo que se buscaba era comparar el uso de los extranjerismos, préstamos adaptados y calcos semánticos identificados en los documentos en gallego del primer corpus, concretamente con los de los artículos de investigación, con su uso en las versiones en castellano de esos mismos artículos. En este caso, la comparación de los textos se realizó de manera manual, dado que una vez localizados los términos en el artículo en gallego, su búsqueda en el artículo correspondiente podía realizarse con facilidad sin ayuda de software específico. Sin embargo, para contrastar los resultados, se procedió a procesar las versiones en castellano de los artículos de manera independiente con la herramienta Word list de AntConc 3.2.4, con el fin de determinar si se detectaban en dichas versiones tanto otras apariciones de los términos identificados en gallego, como apariciones de otros extranjerismos, préstamos o calcos semánticos que no estuviesen presentes en las versiones gallegas.

\section{Resultados y discusión}

A continuación, presentamos y analizamos los resultados obtenidos a partir de la explotación de los dos corpus compilados en lo que se refiere a la presencia de extranjerismos, préstamos adaptados, calcos semánticos y castellanismos. Para facilitar la lectura de dichos resultados, principalmente los del primer corpus, y su uso como fuente de referencia en el aula de traducción económica, se representan por medio de tablas todas las apariciones de extranjerismos (tabla 2), calcos semánticos (tabla 3), préstamos adaptados (tabla 4) y castellanismos (tabla 5).

\subsection{Extranjerismos}

La tabla 2 muestra todos los extranjerismos localizados en el corpus monolingüe, clasificados por el tipo de texto en el que aparecen, la frecuencia de aparición y la presencia del término en las fuentes de referencia consultadas. Se marcan en negrita los términos que fueron localizados en los dos tipos de textos. En aquellos casos en los que no existe en lengua gallega un equivalente acuñado para sustituir el término, se emplea la indicación 'no aplicable' (N/A) en la casilla correspondiente a la frecuencia de uso. 


\begin{tabular}{|c|c|c|c|c|c|}
\hline \multirow{2}{*}{$\begin{array}{l}\text { Tipo de término } \\
\text { Extranjerismo/ } \\
\text { Equivalente }\end{array}$} & \multicolumn{2}{|c|}{ Textos periodísticos } & \multicolumn{2}{|c|}{ Artículos de revista } & \multirow{2}{*}{$\begin{array}{c}\begin{array}{c}\text { Fuentes de } \\
\text { referencia }\end{array} \\
\begin{array}{c}\text { Fuente } \\
\text { (frecuencia) }\end{array}\end{array}$} \\
\hline & $\begin{array}{l}\text { Frecuencia } \\
\text { del término }\end{array}$ & $\begin{array}{c}\text { Frecuencia } \\
\text { del } \\
\text { equivalente }\end{array}$ & $\begin{array}{l}\text { Frecuencia } \\
\text { del término }\end{array}$ & $\begin{array}{c}\text { Frecuencia } \\
\text { del } \\
\text { equivalente }\end{array}$ & \\
\hline $\begin{array}{l}\text { beneficiary }^{5} / \\
\text { beneficiario }\end{array}$ & 0 & 0 & 4 & 11 & \\
\hline bonus/retribución & 3 & 0 & 2 & 0 & \\
\hline boom/auxe & 86 & 196 & 12 & 4 & $\begin{array}{l}\text { NPG, } \\
\text { CORGA(33) }\end{array}$ \\
\hline broker/comisionista & 1 & 0 & 4 & 0 & $\begin{array}{l}\text { NPG, } \\
\text { CORGA(5), } \\
\text { CTG(1) }\end{array}$ \\
\hline business angels & 0 & N/A & 1 & N/A & \\
\hline $\begin{array}{l}\text { cash flows/fluxos de } \\
\text { caixa }\end{array}$ & 0 & 0 & 3 & 13 & $\begin{array}{l}\text { NPG, } \\
\text { CORGA(14) }\end{array}$ \\
\hline cash surrender value & 0 & N/A & 1 & N/A & \\
\hline confirming & 1 & N/A & 0 & N/A & \\
\hline $\begin{array}{l}\text { credit crunch/crise } \\
\text { de liquidez }\end{array}$ & 3 & 2 & 1 & 1 & \\
\hline dealer/mediador & 4 & 0 & 0 & 0 & \\
\hline $\begin{array}{l}\text { dumping/ } \\
\text { afundimento de } \\
\text { prezos }\end{array}$ & 29 & 0 & 0 & 0 & $\begin{array}{l}\text { NPG, } \\
\text { CORGA(12), } \\
\text { VGE, } \\
\text { CTG(5) } \\
\end{array}$ \\
\hline factoring & 1 & 0 & 0 & 0 & \\
\hline fair market & 0 & N/A & 1 & N/A & \\
\hline gap & 0 & N/A & 1 & N/A & \\
\hline $\begin{array}{l}\text { hedge fund/fondo de } \\
\text { cobertura }\end{array}$ & 3 & 2 & 3 & 0 & \\
\hline $\begin{array}{l}\text { holding/ } \\
\text { conglomerado }\end{array}$ & 49 & 0 & 13 & 12 & $\begin{array}{l}\text { NPG, } \\
\text { CORGA(33), } \\
\text { CLUVI(1), } \\
\text { CTG(3) }\end{array}$ \\
\hline $\begin{array}{l}\text { holdout (fondo } \\
\text { voitre) }\end{array}$ & 8 & N/A & 0 & N/A & \\
\hline input & 2 & N/A & 6 & N/A & CORGA(12) \\
\hline $\begin{array}{l}\text { joint venture/ } \\
\text { empresa temporal }\end{array}$ & 2 & 0 & 3 & 0 & $\mathrm{CTG}(2)$ \\
\hline $\begin{array}{l}\text { leasing/ } \\
\text { arrendamento } \\
\text { financeiro }\end{array}$ & 5 & 6 & 2 & 0 & CORGA(14) \\
\hline $\begin{array}{l}\text { leverage/ } \\
\text { apancamento }\end{array}$ & 0 & 1 & 0 & 103 & \\
\hline
\end{tabular}

5. Empleado en conexión con trust. Se recoge la frecuencia de beneficiario en gallego cuando se utiliza en contextos similares.

6. Véase tabla 3: calcos semánticos. 


\begin{tabular}{|c|c|c|c|c|c|}
\hline management/xestión & 3 & 3 & 0 & 99 & \\
\hline matching & 0 & N/A & 1 & N/A & \\
\hline $\begin{array}{l}\text { mobbing/acoso } \\
\text { psicolóxico }\end{array}$ & 146 & 0 & 88 & 20 & $\operatorname{CORGA}(2)$ \\
\hline $\begin{array}{l}\text { offshore/ } \\
\text { extraterritorial, con } \\
\text { vantaxes fiscais, } \\
\text { inscrita nun paraíso } \\
\text { fiscal, no estranxeiro }\end{array}$ & 1 & 0 & 15 & 0 & \\
\hline output & 2 & N/A & 2 & N/A & CORGA(16) \\
\hline output gap & 0 & N/A & 2 & N/A & \\
\hline parent/matriz & 0 & 0 & 4 & 7 & \\
\hline préstamo bullet & 1 & N/A & 0 & N/A & \\
\hline rating/cualificación & 26 & 9 & 5 & 0 & $\begin{array}{l}\text { CORGA(4), } \\
\text { CLUVI(5), } \\
\text { CTG(3) }\end{array}$ \\
\hline renting & 4 & N/A & 0 & N/A & \\
\hline safe havens & 0 & N/A & 1 & N/A & \\
\hline setlor & 0 & N/A & 2 & N/A & \\
\hline shock & 0 & N/A & 31 & N/A & \\
\hline soft law & 0 & N/A & 3 & N/A & \\
\hline spin-off & 21 & N/A & 2 & N/A & CORGA(13) \\
\hline spin-out & 0 & N/A & 1 & N/A & \\
\hline $\begin{array}{l}\text { stakeholder/grupo de } \\
\text { interese }\end{array}$ & 0 & 3 & 30 & 9 & \\
\hline stock/reservas & 6 & 2 & 12 & 2 & CORGA(56) \\
\hline $\begin{array}{l}\text { subprime/baixa } \\
\text { calidade, alto risco }\end{array}$ & 24 & 1 & 3 & 1 & CORGA(1) \\
\hline swap/permuta & 8 & 0 & 9 & 3 & $\begin{array}{l}\text { CORGA(3), } \\
\text { CLUVI(1), } \\
\text { CTG }(7)\end{array}$ \\
\hline $\begin{array}{l}\text { tax lease/ } \\
\text { arrendamento fiscal }\end{array}$ & 71 & 0 & 0 & 0 & \\
\hline treaty shopping & 0 & N/A & 5 & N/A & \\
\hline $\begin{array}{l}\text { trust/fideicomiso - } \\
\text { fondo fiduciario }\end{array}$ & 3 & 0 & 26 & 4 & CORGA(7) \\
\hline $\begin{array}{l}\text { trustee / } \\
\text { fideicomisario }\end{array}$ & 0 & 0 & 4 & 0 & \\
\hline
\end{tabular}

Tabla 2: Extranjerismos localizados en el corpus 1 por orden de frecuencia de aparición

Como se puede observar (gráfico 1), la presencia de extranjerismos es más abundante en los artículos de investigación, en los que se registró la presencia de 35 de los 45 términos detectados, frente a los 27 registrados en las noticias de prensa. Sin embargo, las repeticiones tienden a ser más frecuentes en las noticias de prensa (gráfico 2), más si tenemos en cuenta que, en algunos 


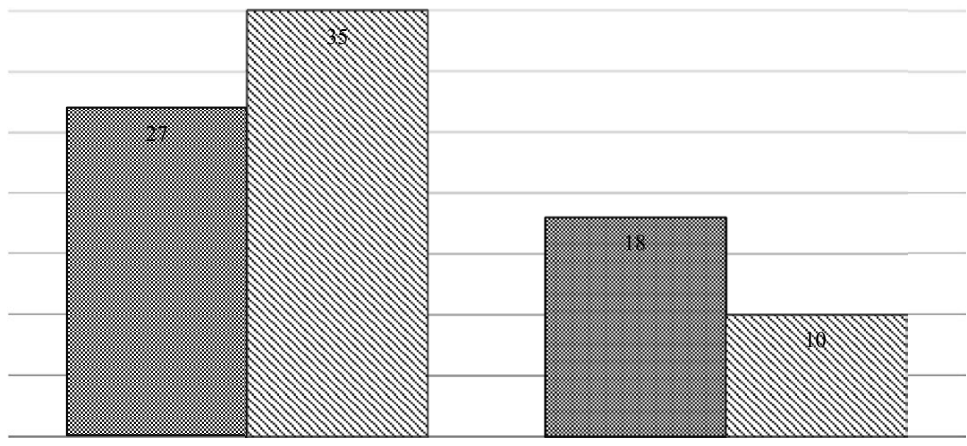

localizados

no localizados

@extranjerismos - prensa \$extranjerismos - artículos de investigación

Gráfico 1: Presencia global de extranjerismos por tipo de texto
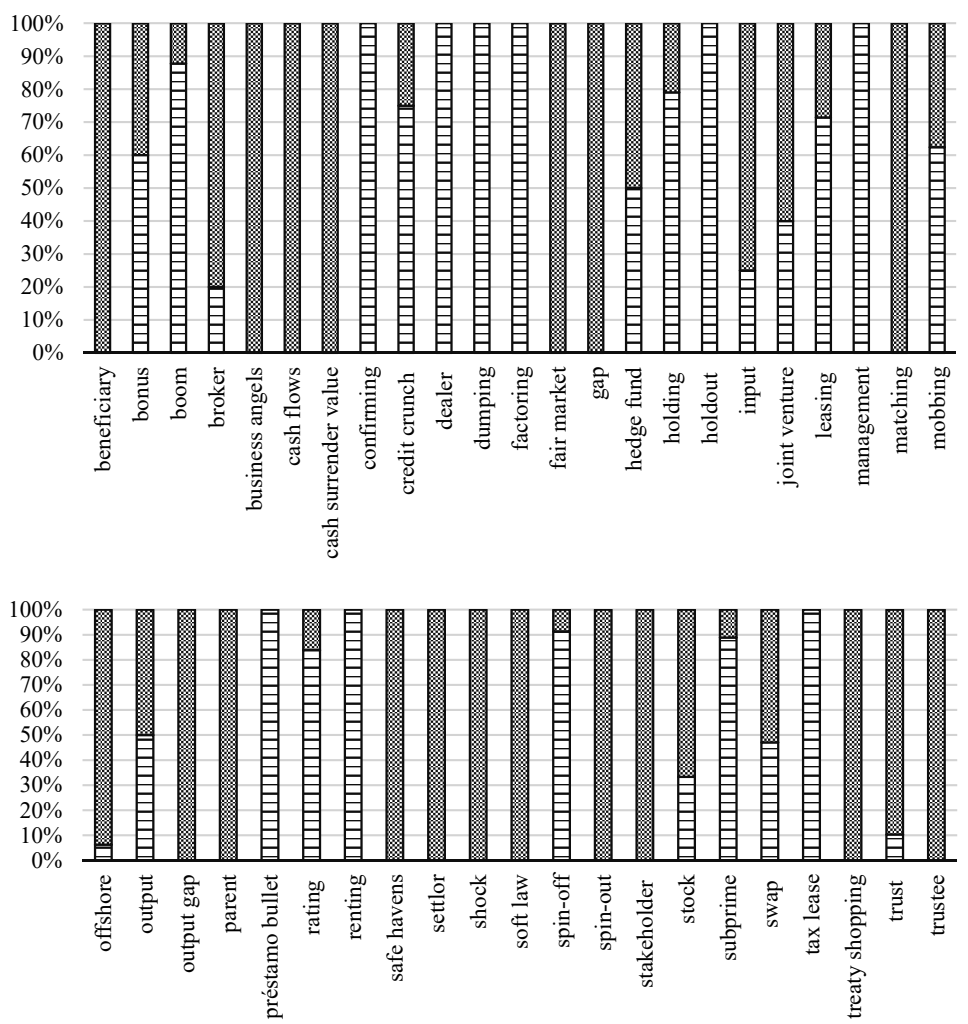

Gráfico 2: Repeticiones de extranjerismos por tipo de texto 
casos, las repeticiones en los artículos de investigación corresponden a un mismo texto.

Como ya se indicó, los términos marcados en negrita en la tabla, 18 de los 45 totales, fueron localizados en ambos tipos de textos. En siete de ellos (bonus, credit crunch, hedge fund, joint venture, leasing, output y swap) no se aprecian diferencias importantes en el número de repeticiones de un tipo de fuente a otra. Seis de los términos (boom, holding, mobbing, rating, spin-off y subprime) presentan un número muy superior de repeticiones en los textos periodísticos frente a los artículos de investigación, mientras que cinco (broker, input, offshore, stock y trust) se emplean con mayor frecuencia en los artículos de investigación. Con todo, como se puede comprobar en la tabla 2, las diferencias de frecuencia son menores en este último grupo de términos.

Los anglicismos con un mayor número de repeticiones en los artículos de investigación (mobbing, shock, trust, stakeholder, offshore, holding, boom y stock) son en su mayor parte neologismos incorporados a la lengua antes de la crisis. Son los extranjerismos asociados con la crisis, cuyo uso se ha popularizado en los últimos años, los que aparecen bien en ambos tipos de textos (hedge fund, credit crunch, swap) bien solo en los artículos periodísticos (rating, subprime, tax lease). En general, la presencia de los extranjerismos es escasa en el CORGA (11) y mínima en el resto de los corpus de referencia consultados ( 5 en NPG, 5 en CTG, 1 en VGE y 2 en CLUVI).

Por lo que se refiere a la distribución de los términos dentro de los textos, en varios casos todas las repeticiones de un extranjerismo se concentran en el mismo artículo de investigación (mobbing, trust, swap). También en los artículos de investigación, los extranjerismos se concentran principalmente en artículos de temática internacional y suelen aparecer explicados o acompañados de una propuesta de traducción, en consonancia con el carácter académico de los artículos (ejemplo 1). En ocasiones, es el término inglés es el que se utiliza para ampliar el término gallego, cuando se considera que no está suficientemente asentado (ejemplos 2 y 3 ). Además, muchos aparecen utilizados de manera indistinta junto al término gallego correspondiente en el mismo artículo: ej. parent /matriz (ejemplos 4 y 5).

Ej. 1: o termo stakeholder ou grupo de interese foi definido por Freeman (1984) como "aquelas persoas, grupos ou organizacións que manteñen unha relación directa ou indirecta coa empresa e que, polo tanto, poden verse afectados polas decisións e accións da organización, e que reciprocamente poden influir ou exercer poder nela" (RGE) (nuestro subrayado).

Ej. 2: Os créditos hipotecarios estadounidenses de baixa calidade (subprime) desenvolvéronse com extraordinaria rapidez debido principalmente á abundancia de crédito derivada do exceso de liquidez mundial (RGE) (nuestro subrayado). 
Ej. 3: Por outro lado, as axencias de cualificación de risco (rating agencies) tampouco fixeron ben o seu traballo e, ademais, seguiron sendo un oligopolio estadounidense (RGE) (nuestro subrayado)

Ej. 4: [...] non podemos, porén, realizar a tradicional análise dun proxecto de investimento internacional tanto desde a óptica local como desde a do parent cando se trata dun proxecto de investimento directo ou real (RGE) (nuestro subrayado).

Ej. 5: [...] os fondos poden ser enviados á empresa matriz, reinvestidos ou transferidos como préstamos ás filiais (RGE) (nuestro subrayado).

Por el contrario, en los textos periodísticos, los extranjerismos se recogen en diferentes noticias y en todas las publicaciones, si bien es cierto que se ha detectado un mayor número en las fuentes más recientes (Praza Pública y Sermos Galiza), salvo en el caso de rating y subprime, que se registran con igual frecuencia en todos los periódicos. Llaman especialmente la atención los términos dumping y holding, los cuales, pese a ser anglicismos ya identificados por Araya \& Gómez Clemente en su estudio de 1999, no se han documentado ni en los textos extraídos de Vieiros ni en los de Galiza Hoxe. ${ }^{7}$ En general, los términos suelen aparecer integrados en el texto sin ningún tipo de definición, ampliación o traducción (ejemplos 6 y 7), y el uso de marcas ortotipográficas con los extranjerismos responde a patrones irregulares incluso dentro de la misma publicación (ejemplos 8-11), lo que indica que no se encuentran totalmente asentados en la lengua y que las publicaciones carecen de criterios unificados para su tratamiento.

Ej. 6: A "medida estrela" do executivo de Bush sería a creación dunha axencia pública que se vai facer cargo das hipotecas subprime que lastran gravemente as entidades financeiras (vieiros.com) (nuestro subrayado).

Ej. 7: A axencia Fitch baixa o rating do Popular, no que se integrou o Pastor (Galicia Hoxe) (nuestro subrayado).

Ej. 8 A CIG oponse á venda de Novagalicia Banco ao fondo Guggeinheim, ao que cualifica como "especulativo" e responsabiliza de ser un dos máximos responsábeis da crise das 'subprime' (Sermos Galiza) (nuestro subrayado).

Ej. 9 [...] poucos meses antes de que estoupara nos USA a crise das hipotecas subprime (Sermos Galiza) (nuestro subrayado). .

Ej. 10 [...] a pesar das rebaixas de nota por parte das axencias de 'rating' (Galicia Hoxe) (nuestro subrayado).

7. Esta ausencia no parece corresponderse con el uso del término en la actualidad, dado que una consulta a través de la herramienta de búsqueda del sitio web de Galiza Hoxe permitió recuperar 113 noticias en las que se empleaba el término holding y 137 en las que se utilizaba el término dumping, si bien las más antiguas se remontan a 2007 y la mayor parte de ellas databan de después de 2011. Ello podría ser indicador de un cambio en los patrones de incorporación de extranjerismos en los últimos años, algo que debería confirmarse con un estudio diacrónico más específico. 
Ej. 11 [...] que non se conceda a unha axencia de rating o privilexio de determinar se a decisión do Eurogrupo é un éxito ou un fracaso (Galicia Hoxe) (nuestro subrayado).

Por lo que respecta a la coexistencia del extranjerismo y su equivalente en gallego (en aquellas ocasiones en que existe y se ha documentado su presencia), los datos extraídos del corpus indican que, en los textos periodísticos (gráfico 3), en general el uso del extranjerismo prevalece por encima del equivalente gallego, salvo en los casos de los términos credit crunch, un término que podemos considerar relativamente técnico, y boom, cuya frecuencia de uso es inferior a auxe. Destaca, principalmente, el uso de mobbing frente a acoso psicolóxico o acoso laboral, holding frente a conglomerado, tax lease frente a arrendamento fiscal, rating frente a calificación, dumping frente a afundimento de prezos y subprime frente a lixo.

En los artículos de investigación (gráfico 4), por el contrario, se registran más casos en los que se utiliza con casi igual o mayor frecuencia el término equivalente (fluxos de caixa frente a cash flow, conglomerado frente a holding, matriz frente a parent) y casos en los que no se registra el uso del extranjerismo y sí del equivalente (xestión frente a management, apancamento frente a leverage). Con todo, se registran también casos en que el extranjerismo prevalece por encima del equivalente (trust frente a fideicomiso, swap frente a permuta,

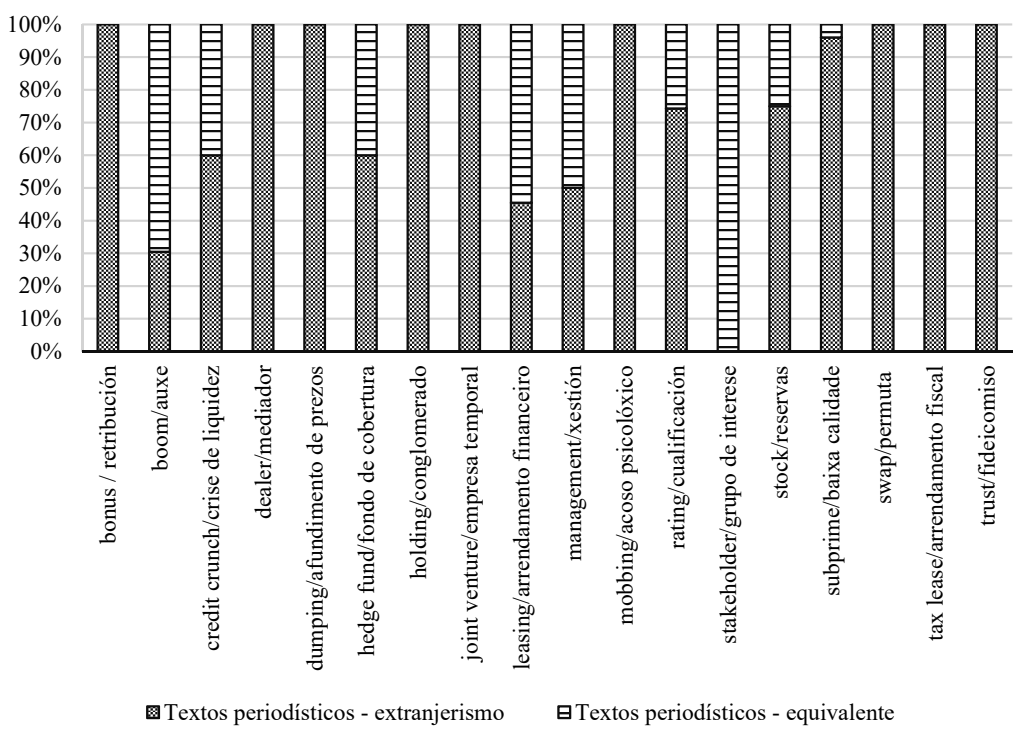

Gráfico 3: Coexistencia de extranjerismo y equivalente en textos periodísticos 


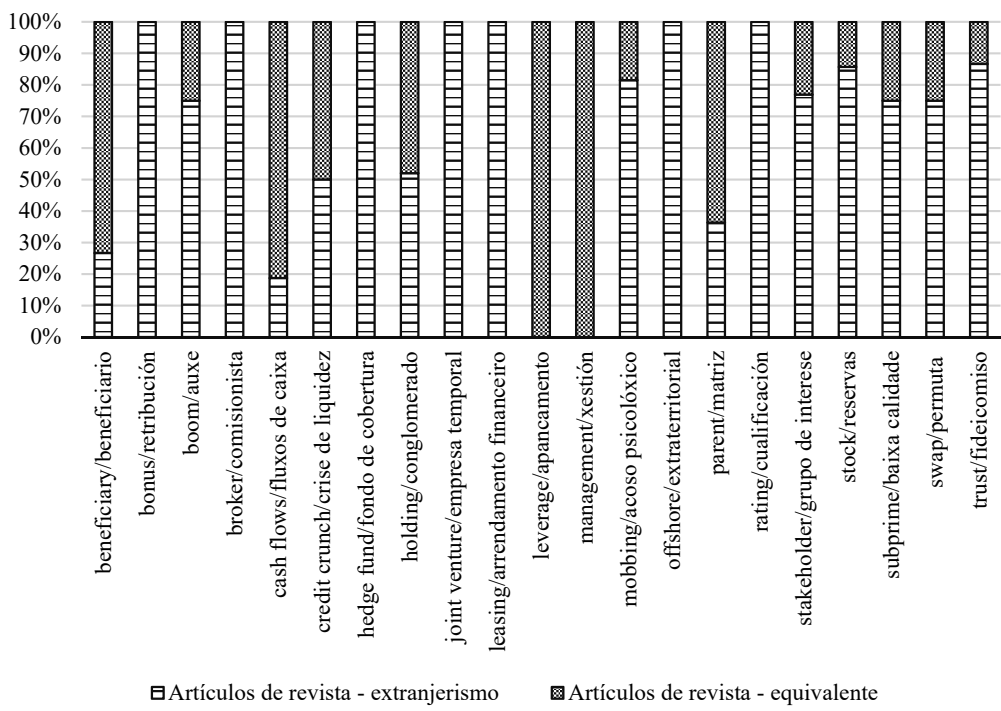

Gráfico 4: Coexistencia de extranjerismo y equivalente en artículos de revista

stock frente a reservas), incluso en casos en los que podríamos considerar que se trata de extranjerismos innecesarios (stakeholder frente a grupos de interese, hedge fund frente a fondo de cobertura, offshore frente a extraterritorial, libre de impostos, en paraíso fiscal, etc.)

\subsection{Calcos semánticos}

Aunque la presencia de calcos semánticos (tabla 3) es similar en ambos tipos de textos, los de mayor frecuencia, los relacionados con la crisis, presentan más repeticiones en los textos periodísticos. La mayor parte de los calcos consultados se han documentado en el CORGA, que cubre textos hasta 2014, lo que indica que se trata de términos utilizados en la lengua, pero son pocos los que aparecen en el resto de fuentes de referencia, probablemente debido a la antigüedad de estas. Así sucede, por ejemplo, con los términos fondo voitre, bono lixo, banco malo y activo tóxico, voces que aparecen en los textos periodísticos y no se recogen en los artículos de investigación. También es el caso de prima de risco, la voz con mayor frecuencia de uso de todas las analizadas, cuya aparición solamente se ha registrado una vez en el corpus técnico del gallego, y ninguna en el CLUVI, en el VGE ni en el NPG. Aunque burbulla y rescate podrían considerase excepciones a esta tendencia, ya que sí aparecen en el CTG (burbulla también en el VGE), hemos de tener en cuenta que se trata de voces generales de la lengua que han ampliado su significado, lo que 
Traducción económica al gallego: pautas de actuación ante la presencia de...

\begin{tabular}{|c|c|c|c|c|c|}
\hline \multirow[b]{2}{*}{$\begin{array}{c}\text { Tipo de término } \\
\text { Calco }\end{array}$} & \multicolumn{2}{|c|}{ Texto periodístico } & \multicolumn{2}{|c|}{ Artículo de revista } & \multirow{2}{*}{$\begin{array}{c}\text { Referencias } \\
\begin{array}{c}\text { Fuente } \\
\text { (frecuencia) }\end{array}\end{array}$} \\
\hline & $\begin{array}{l}\text { Frecuencia } \\
\text { del término }\end{array}$ & $\begin{array}{c}\text { Frecuencia } \\
\text { del } \\
\text { equivalente }\end{array}$ & $\begin{array}{l}\text { Frecuencia } \\
\text { del término }\end{array}$ & $\begin{array}{c}\text { Frecuencia } \\
\text { del } \\
\text { equivalente }\end{array}$ & \\
\hline acordo marco & 7 & $N / A^{8}$ & 1 & N/A & $\begin{array}{l}\text { CORGA(23); } \\
\text { NPG }\end{array}$ \\
\hline $\begin{array}{l}\text { activo tóxico/ } \\
\text { deteriorado }\end{array}$ & 35 & 1 & 0 & 1 & CORGA(19) \\
\hline alcista & 0 & N/A & 6 & N/A & $\begin{array}{l}\text { CORGA(35), } \\
\text { CLUVI(2), } \\
\text { CTG(44) } \\
\end{array}$ \\
\hline apancamento & 0 & N/A & 10 & N/A & CTG(1) \\
\hline baixista & 0 & N/A & 1 & N/A & $\begin{array}{l}\text { CORGA(17), } \\
\text { CLUVI(2), } \\
\text { CTG(8) } \\
\end{array}$ \\
\hline $\begin{array}{l}\text { bancarrota/ } \\
\text { quebra }\end{array}$ & 26 & 106 & 0 & 16 & $\begin{array}{l}\text { CORGA(40), } \\
\text { VGE, CTG(1) }\end{array}$ \\
\hline banco malo & 26 & N/A & 0 & N/A & CORGA(8) \\
\hline $\begin{array}{l}\text { bono/hipoteca } \\
\text { lixo }\end{array}$ & 10 & N/A & 1 & N/A & $\begin{array}{l}\text { CORGA(4), } \\
\text { CTG(1) }\end{array}$ \\
\hline burbulla & 56 & N/A & 24 & N/A & $\begin{array}{l}\text { CORGA(76), } \\
\text { VGE, CTG(14) }\end{array}$ \\
\hline capital risco & 23 & N/A & 1 & N/A & \begin{tabular}{|l|} 
CORGA(49), \\
CLUVI(16), \\
CTG(10) \\
\end{tabular} \\
\hline fondo voitre & 12 & N/A & 0 & N/A & \\
\hline liña de crédito & 17 & N/A & 0 & N/A & $\begin{array}{l}\text { CORGA(11), } \\
\text { CLUVI(1), } \\
\text { CTG(2) }\end{array}$ \\
\hline OPA & 11 & N/A & 0 & N/A & CORGA(90) \\
\hline prima de risco & 155 & N/A & 11 & N/A & $\begin{array}{l}\text { CORGA(22), } \\
\text { CTG(1) }\end{array}$ \\
\hline rescate & 69 & N/A & 8 & N/A & $\begin{array}{l}\text { CORGA(319), } \\
\text { CTG(41) } \\
\end{array}$ \\
\hline risco cambiario & 9 & N/A & 16 & N/A & \begin{tabular}{|l} 
VGE, \\
CLUVI(2) \\
\end{tabular} \\
\hline titulización & 0 & N/A & 10 & N/A & $\begin{array}{l}\text { CORGA(4), } \\
\text { CLUVI(5), } \\
\text { CTG(20) } \\
\end{array}$ \\
\hline valor nominal & 4 & N/A & 3 & N/A & $\begin{array}{l}\text { CORGA(10), } \\
\text { Vg2, } \\
\text { CLUVI(6), } \\
\text { CTG(22) }\end{array}$ \\
\hline
\end{tabular}

Tabla 3: Calcos semánticos localizados en el corpus 1 ordenados por frecuencia de aparición

8. Al igual que en la tabla 2, en aquellos casos en los que no existe en lengua gallega un equivalente acuñado para sustituir el término, se emplea la indicación 'no aplicable' (N/A) en la casilla correspondiente a la frecuencia de uso. 
justifica su presencia en las fuentes de referencia. Con todo, los datos relativos a su presencia en el CORGA, sección economía, (76 para burbulla y 319 para rescate), indica que son préstamos utilizados con frecuencia en textos de temática económica. Los dos términos más técnicos registrados, apancamento y titulización, por su parte, solo se utilizan en los artículos de investigación, lo que resulta coherente con el tipo de texto y el destinatario del mismo.

Solo en dos casos (bancarrota y activo tóxico) se ha constado la presencia de un equivalente autóctono del término en el corpus, con tratamientos diferentes. Así, el término bancarrota, que solo se ha identificado en textos periodísticos, aparece sustituido en muchos casos por el término quebra o creba, que es el único documentado en los artículos de investigación. Por el contrario, el término activo tóxico se emplea preferentemente frente a la opción activo deteriorado, cuyo uso solo se registra una vez en los textos periodísticos y otra en los artículos de investigación.

\subsection{Préstamos adaptados}

La presencia de préstamos adaptados en los textos del corpus es muy escasa (tabla 4) y los pocos que se han podido registrar se corresponden con términos

\begin{tabular}{|c|c|c|c|c|c|}
\hline \multirow[b]{2}{*}{$\begin{array}{l}\text { Tipo de término } \\
\text { Préstamo adaptado }\end{array}$} & \multicolumn{2}{|c|}{ Texto periodístico } & \multicolumn{2}{|c|}{ Artículo de revista } & \multirow{2}{*}{$\begin{array}{c}\text { Referencias } \\
\text { Fuente }^{9}\end{array}$} \\
\hline & $\begin{array}{l}\text { Frecuencia } \\
\text { del término }\end{array}$ & $\begin{array}{c}\text { Frecuencia } \\
\text { del } \\
\text { equivalente }\end{array}$ & $\begin{array}{l}\text { Frecuencia } \\
\text { del término }\end{array}$ & $\begin{array}{c}\text { Frecuencia } \\
\text { del } \\
\text { equivalente }\end{array}$ & \\
\hline absentismo & 1 & N/A & 1 & N/A & RAG \\
\hline inflación & 42 & N/A & 57 & N/A & RAG \\
\hline deflación & 22 & N/A & 4 & N/A & RAG \\
\hline auditoría & 14 & N/A & 4 & N/A & RAG \\
\hline boicot & 1 & N/A & 0 & N/A & RAG \\
\hline consultoría & 5 & N/A & 3 & N/A & RAG \\
\hline status/estatus ${ }^{10}$ & 1 & 1 & $4 / 4$ & $7 / 7$ & $\begin{array}{l}\text { RAG/CORGA } \\
(76) \text { CTG } \\
(100), \text { CLUVI } \\
(1),\end{array}$ \\
\hline
\end{tabular}

Tabla 4: Préstamos adaptados localizados en el corpus 1 por orden de frecuencia de aparición

9. Al tratarse de términos ya asentados en la lengua gallega, se han encontrado todos en el Dicionario da Real Academia Galega, razón por la cual no se ha determinado su presencia en otras fuentes de referencia, salvo en el caso de estatus (véase nota 10).

10. Anglicismo de origen latino. El Dicionario da Real Academia Galega reconoce la forma sin adaptar, pero no la forma adaptada. Sin embargo, sí se recoge la forma estatus en el 
en general muy asentados en la lengua y que, por lo tanto, podríamos reconocer como préstamos pero no como neologismos.

\subsection{Castellanismos}

Pese a que, estrictamente, los castellanismos podrían considerase extranjerismos, los resultados de nuestro estudio nos indican que en su mayoría constituyen, tal como indicaban Araya \& Gómez (1999: 135) interferencias por desconocimiento de las soluciones autóctonas. Por ese motivo, se ha tomado la decisión de presentarlos de manera aislada, en lugar de fusionarlos con los extranjerismos.

Tal y como se muestra en la tabla 5, a excepción del término plantilla en lugar de cadro de persoal, que se detectó en un artículo de investigación, solo se detectaron castellanismos en los textos periodísticos. La búsqueda específica de castellanismos habituales en el lenguaje económico gallego, como son presuposto (orzamento), apalancamiento (apancamento), inversor ('investidor'), devengo (devindicación) o intereses (xuros) no arrojó ninguna coincidencia en los artículos de investigación, lo que puede explicarse por el proceso de revisión al que se someten los trabajos en gallego (sean originales sean traducciones) antes de su publicación. Tres de los castellanismos inicialmente seleccionados, bursátil, rentabilidade y subasta, no se registraron en ninguno de los dos tipos de textos, mientras que otros, como calificación o venta, registraron un número de apariciones muy bajo. Por último, la frecuencia de uso del término correcto gallego es más frecuente en todos los casos y se registró su uso tanto en prensa como en artículos de investigación para todos ellos con excepción de apancamento, utilizado solo en el segundo tipo de textos.

Una excepción destacable la constituye el castellanismo devengar, el único registrado frente al término gallego devindicar, cuyo uso no se ha podido registrar en ningún tipo de texto. Su ausencia, sin embargo, se debe probablemente a que se trata de un neologismo incorporado a los diccionarios en 2011 (RAG, en línea), mientras que devengar y devengo, pese a haber tenido históricamente la consideración de castellanismos, son las formas empleadas en muchos textos legales traducidos del castellano al gallego, entre ellos los Planes Generales de Contabilidad de 1990 y 2007.

CORGA, en el CTG y en el CLUVI, y en el corpus se han documentado ambas, con una ligera diferencia a favor de la forma adaptada en los artículos de investigación. 


\begin{tabular}{|c|c|c|c|c|c|}
\hline Castellanismo $^{11}$ & $\begin{array}{l}\text { Término } \\
\text { gallego }\end{array}$ & $\begin{array}{l}\text { Frecuencia del } \\
\text { castellanismo }\end{array}$ & $\begin{array}{c}\text { Frecuencia } \\
\text { del término } \\
\text { gallego }\end{array}$ & $\begin{array}{c}\text { Fuente del } \\
\text { castellanismo }\end{array}$ & $\begin{array}{c}\text { Fuente del } \\
\text { término } \\
\text { gallego }\end{array}$ \\
\hline *intereses & xuros & 18 & 193 & Periódico & $\begin{array}{l}\text { Periódico/ } \\
\text { Artículo }\end{array}$ \\
\hline *apalancamiento & apancamento & 1 & 10 & Periódico & Artículo \\
\hline presuposto & orzamento & 7 & 120 & Periódico & $\begin{array}{l}\text { Periódico/ } \\
\text { Artículo }\end{array}$ \\
\hline $\begin{array}{l}\text { inversor/ } \\
\text { inversión/ }\end{array}$ & $\begin{array}{l}\text { investidor/ } \\
\text { investimento }\end{array}$ & 4 & 347 & Periódico & $\begin{array}{l}\text { Periódico/ } \\
\text { Artículo }\end{array}$ \\
\hline $\begin{array}{l}\text { devengar/ } \\
\text { devengo }\end{array}$ & $\begin{array}{l}\text { devindicar/ } \\
\text { devindicación }\end{array}$ & 6 & 0 & Periódico & N/A \\
\hline *venta & venda & 4 & 57 & Periódico & $\begin{array}{l}\text { Periódico/ } \\
\text { Articulo }\end{array}$ \\
\hline *bursátil & bolsista & 0 & 4 & N/A & $\begin{array}{l}\text { Periódico/ } \\
\text { Articulo }\end{array}$ \\
\hline calificación & cualificación & 2 & 98 & Periódico & $\begin{array}{l}\text { Periódico/ } \\
\text { Articulo }\end{array}$ \\
\hline${ }^{*}$ producto & produto & 6 & 398 & Periódico & $\begin{array}{l}\text { Periódico/ } \\
\text { Articulo }\end{array}$ \\
\hline pago & pagamento & 9 & 54 & Periódico & $\begin{array}{l}\text { Periódico/ } \\
\text { Articulo }\end{array}$ \\
\hline${ }^{*}$ plantilla & $\begin{array}{l}\text { cadro de } \\
\text { persoal }\end{array}$ & 1 & 9 & Artículo & $\begin{array}{l}\text { Periódico/ } \\
\text { Articulo }\end{array}$ \\
\hline *rentabilidade & rendibilidade & 0 & 49 & N/A & $\begin{array}{l}\text { Periódico/ } \\
\text { Articulo }\end{array}$ \\
\hline${ }^{*}$ subasta & poxa & 0 & 4 & N/A & $\begin{array}{l}\text { Periódico/ } \\
\text { Articulo }\end{array}$ \\
\hline
\end{tabular}

Tabla 5: Castellanismos localizados en el corpus 1 por orden de frecuencia de aparición

Dentro de los textos periodísticos, los castellanismos son igual de frecuentes en los que son traducciones de textos redactados originariamente en castellano (Galicia Hoxe), que en los aparentemente redactados en gallego. Cabría analizar en qué medida esto es debido a la práctica de la traducción encubierta, todavía muy arraigada en ciertos sectores de la vida pública gallega, como ya se indicó en el apartado 3.1.

\subsection{Análisis del corpus bilingüe}

Para analizar el segundo corpus, se partió del listado de extranjerismos, préstamos adaptados y calcos semánticos registrados en los artículos de

11. Se marcan en la tabla con asterisco aquellos castellanismos que no están aceptados como correctos en el Dicionario da Real Academia Galega. 
investigación en lengua gallega del primer corpus y se comprobó cada una de las apariciones en las versiones castellanas de los mismos artículos. La búsqueda de más repeticiones de los términos, así como de términos no registrados en los artículos en gallego fue igualmente infructuosa. Esta circunstancia parece estar en contradicción con la constatación de Baker (2004: 172), respecto a la tendencia hacia un uso más conservador del lenguaje en la traducción y confirma nuestra hipótesis (cfr. apartado 3.1) de que dicha tendencia podría no darse en el caso de lenguas estructuralmente muy próximas, como son el castellano y el gallego. Ha de tenerse en cuenta el hecho de que, como se acaba de indicar, la coincidencia fue del $100 \%$, lo que descarta la posibilidad de que las coincidencias se localizasen únicamente en casos de autotraducción, mientras que las traducciones realizadas por un tercero se apartasen de esta práctica. Con todo, se considera necesario realizar un estudio detallado con un corpus más extenso de originales y traducciones compuesto por tipos de textos diferentes, ya que la procedencia de todos los textos de una misma publicación puede haber contribuido a sesgar los resultados.

\section{Conclusiones}

Tras la compilación y revisión de los corpus, seguidas del análisis de los datos obtenidos, formularemos a continuación una serie de conclusiones preliminares que, necesariamente, deberán ser corroboradas con estudios más amplios tanto en número de palabras como en tipos de textos estudiados.

En primer lugar, por lo que se refiere a los mecanismos de incorporación de neologismos a la lengua gallega, pese a la importancia del neologismo de préstamo en las lenguas de especialidad, los resultados no permiten afirmar que haya superado al de forma en la incorporación de neologismos a la lengua gallega. Además, teniendo en cuenta los resultados del análisis del segundo corpus, así como los resultados de trabajos previos (Orts \& Almela 2009; Orts $\&$ Almela 2012), no se detectan diferencias respecto a las pautas de incorporación de términos económicos en lengua española.

Por lo que se refiere a las tendencias por tipos de textos y su utilidad para los estudiantes de traducción, los resultados parecen indicar que la utilización de extranjerismos en los artículos de investigación responde a una decisión consciente del autor, que en ocasiones los utiliza de manera indistinta con el término equivalente y en muchos casos de manera complementaria. Esta utilización se perpetúa en la versión traducida del texto, tanto si se trata de autotraducción, como en artículos traducidos por la propia revista. Ello permite confirmar este uso consciente de los términos y la probabilidad de que los autores no viesen con agrado el hecho de que una traducción realizada por 
terceros no respetase este uso. Con todo, la presencia de extranjerismos en este tipo de textos se ha revelado como poco frecuente en términos generales, por lo que los estudiantes y futuros traductores deben intentar limitar su uso a aquellos casos en que se trate de voces muy especializadas o que carezcan de un equivalente exacto. Por otra parte, el uso de los extranjerismos en los artículos de investigación acompañados de propuestas de traducción convierte los artículos en una importante fuente de referencia, no solo informativa sino también terminológica, que los estudiantes de traducción deberían tener en cuenta frente a fuentes tradicionales menos productivas como diccionarios y glosarios en otras lenguas o textos extraídos de internet.

Frente al uso que de ellos se hace en los artículos de investigación, la presencia de extranjerismos y calcos semánticos en los artículos de prensa responde a la premura por elaborar el texto o al desconocimiento de la existencia de un término gallego equivalente asentado. No se utilizan extranjerismos cuando existen términos gallegos ya acuñados, como es el caso de matriz frente a parent. En este sentido, y aunque la fiabilidad de los textos periodísticos como fuente de referencia es baja, por tratarse de textos redactados por lo general por personas no expertas en economía, ha de tenerse en cuenta que constituyen un reflejo de la lengua a la que están expuestos los no expertos en materia económica. Por este motivo, su utilización como fuentes de referencia no es descartable cuando se traduzcan textos informativos o de divulgación dirigidos a no expertos.

Por otra parte, desde el punto de vista metodológico, la sencillez de la metodología empleada la convierte en una herramienta de gran utilidad en el aula de traducción económica inglés-gallego. En concreto, su aplicación podría permitir a los estudiantes reflexionar en primera persona sobre la pertinencia de utilizar o no un término en función de los resultados obtenidos a partir de su propia búsqueda, en lugar de basarse en fuentes de referencia indirectas en lengua española o portuguesa, práctica habitual en el contexto de la traducción económica hacia la lengua gallega. Aunque se han realizado ya algunas experiencias didácticas en este sentido, los resultados exceden los objetivos de este estudio, por lo que serán objeto de trabajos futuros.

Con todo, de cara al futuro, se revela necesaria una ampliación y diversificación de los dos corpus utilizados, que permita ratificar o contrastar las conclusiones necesariamente preliminares aquí recogidas. En concreto, se plantea la ampliación del corpus de artículos de investigación a partir de trabajos publicados en otras fuentes, así como la ampliación retrospectiva y actualización continua del corpus de textos periodísticos, para medir la evolución de la terminología económica experimentada en los años pasados y la que se vaya 
produciendo en los próximos años, en un momento en el que la presencia de noticias sobre la crisis económica en la prensa parece ir atenuándose.

\section{Referencias bibliográficas}

ARAYA, Raúl \& Xosé María Gómez Clemente. (1999) "Aspectos lingüísticos da neoloxía na lingua galega." En: López, Ana Cristina \& Cristina Martins (eds.) 1999. Actas do XIV Encontro Nacional da Associação Portuguesa de Lingüística (Aveiro, 28-29 de septiembre de 1998). 2 vols. Braga: Associação Portuguesa de Lingüística, pp. 135-160.

AUgER, Pierre \& Louis Jean Rousseau. (1978) Méthodologie de la recherche terminologique. Quebec: Office de la langue française.

BAKER, Mona. (2004) "A corpus-based view of similarity and difference in translation." International Journal of Corpus Linguistics 9:2, pp. 167-193.

BAKER, Mona. (1995) "Corpora in Translation Studies." Target 7:2, pp. 223-243.

BOWKER, Lynne \& Jennifer Pearson. (2002) Working with Specialized Language: a practical guide to using corpora. London: Routledge.

CABRE, María Teresa; Judit Freixa \& Elisabet Solé. (1999) "À la limite des mots construits possibles." En: Corbin, Danielle et al. (eds.) 1999. Mots possibles et mots existants. Forum de morphologie. Actes du Colloque de Villeneuve de Ascq (28-29 avril 1997), publications de l'U.R.A., 382 du CNRS (SILEX), Université de Lille III, pp. 65-78.

CORPAS, Gloria. (2001) "Compilación de un corpus ad hoc para la enseñanza de la traducción inversa especializa." Trans 5, pp. 155-184.

Crystal, David. (2011) "The Consequences of Global English." En: PowellDavis, Phillip (ed.) 2011. Word for Word: The social, economic and political impact of Spanish and English. Madrid: Santillana, Instituto Cervantes \& the British Council, pp. 67-72.

FIEDLER, Sabine. (2011) "The Sky is the Limit: The Influence of English on German Phraseology." En: Szerszunowicz, Joanna; Boguslaw Nowowiejski; Yagi Katsumasa \& Kanzaki Takaaki (eds.) 2011. Focal Issues of Phraseological Studies. Bialystok: University of Bialystok Publishing House, pp. 247-268.

FURIASSI, Cristiano; Virginia Pulcini \& Félix Rodríguez González. (2012) "The lexical influence of English on European languages: From words to phraseology." En: Furiassi, Cristiano; Virginia Pulcini \& Félix Rodríguez González (eds.) 2012. The Anglicization of European Lexis. Amsterdam \& Filadelfia: John Benjamins, pp. 1-24.

GarCía GONZÁLEZ, Marta. (2013) Adaptaciones lingüisticas y culturales en la localización de sitios web como elementos clave en la estrategia de internacionalización empresarial. Vigo: Universidade de Vigo. Trabajo de fin de máster inédito. 
García GonzÁlez, Marta. (2002) "El paper de la traducció en la normalització de la llengua gallega." En: Diaz Fouces, Oscar; Joan Costa Carreras \& Marta García González (eds.) 2002. Traducció i dinámica sociolingüística. Barcelona: Llibres de l'index, pp. 55-84.

Guerrero Ramos, Gloria. (1997) Neologismos en el español actual. Madrid: Arco Libros.

GimÉnEZ, Eduardo Luis \& Francisco Xavier Lores. (2005) Vocabulario de economía. Vigo: Universidade de Vigo.

House, Julianne. (1981) A Model for Translation Quality Assessment. Second Edition. Tübingen: Narr.

Hunston, Susan. (2002) Corpora in Applied Linguistics. Cambridge: Cambridge University Press.

Kennedy, Graeme. (1998) An Introduction to Corpus Linguistics. London: Longman.

LÓPEZ FERNÁNDEZ, Susana et al. (2005) Novas palabras galegas: repertorio de creacións léxicas rexistradas na prensa e internet. Vigo: Universidade de Vigo.

Martí SolAnO, Ramón. (2012) "Multi-word Loan Translations and Semantic Borrowing." En: En Furiassi, Cristiano; Virginia Pulcini \& Félix Rodríguez González (eds.) 2012. The Anglicization of European Lexis. Amsterdam \& Filadelfia: John Benjamins, pp. 199-216.

Nogueira, Alba. (2013) Investigación e lingua: Propostas para a mellora do galego na actividade científica. Vigo: Universidade de Vigo.

OnCíns MARTíneZ, José Luis. (2009) "Towards a Corpus-Based Analysis of Anglicisms in Spanish: A Case Study." International Journal of English Studies, special issue, pp. 115-132.

OnCÍNS MARTínEZ, José Luis. (2012) "Newly-Coined Anglicisms in Contemporary Spanish.” En: Furiassi, Cristiano; Virginia Pulcini \& Félix Rodríguez González (eds.) 2012. The Anglicization of European Lexis. Amsterdam \& Filadelfia: John Benjamins, pp. 217-238.

OnCÍNS MARTíneZ, José Luis. (2014) "English Idioms Borrowed and Reshaped: The Emergence of a Hybrid Metaphor in Spanish." En: Mussolf, Andreas; Fiona MacArthur \& Julio Pagani (eds.) 2014. Metaphor and Intercultural Communication. London: Bloomsbury, pp. 149-155.

ORTS, María Ángeles \& Ángela Almela. (2009) "Translating the Spanish Economic Discourse of the Crisis: Dealing with the Inevitability of English Loanwords." International Journal of English Studies: Recent and Applied Corpus-Based Studies, número especial, pp. 133-157.

OrTS, María Ángeles \& Ángela Almela. (2012) "Deep into the discourse of the Spanish crisis: The deployment of English lexical incorporations to translate the untranslatable." Ibérica: Revista de la Asociación Europea de Lenguas para Fines Específicos 23, pp. 89-108. 
Pearson, Jennifer. (1998) Terms in Context. Amsterdam: John Benjamins.

Rodríguez, Anair \& Montserrat Davila Ventura. (2004) Lingua galega: dúbidas lingüísticas. Vigo: Universidade de Vigo.

RODRíGUEZ GONZÁLEZ, Félix. (2004) "Calcos y traducciones del inglés en español actual." En: Fuentes Olivera, Pedro Antonio (ed.) 2004. Lengua y sociedad: Investigaciones recientes en lingüística aplicada. Valladolid: Universidad de Valladolid, pp. 177-192.

Rozumko, Agata. (2012) "English Influence on Polish Proverbial Language." En: Furiassi, Cristiano; Virginia Pulcini \& Félix Rodríguez González (eds.) 2012. The Anglicization of European Lexis. Amsterdam \& Filadelfia: John Benjamins, pp. 261-280.

Russo, Adriana. (2002) "Préstamos del inglés en la jerga económica: ¿Es posible evitar el sobreendeudamiento lingüístico?" En: Varios autores. 2002. Primer Congreso Internacional: "El español, lengua de traducción". Ciudad Real: Esletra, Comisión Europea \& Agencia EFE, pp. 186-201.

SÁnCHEZ Gijón, Pilar. (2002). "Aplicaciones de la lingüística de corpus a la práctica de traducción." Terminologie et Traduction 2, pp. 84-106.

SinClair, John. (1991) Corpus Concordance Collocation. Oxford: Oxford University Press.

TAPpolet, Ernst. (1913) Die alemannischen Lehnwörter in den Mundarten der französischen Schweiz: eine kulturhistorisch-linguistische Untersuchung. Basel: Universitäts-Buchdruckerei Friedrich Reinhardt.

TORRUELLA, Joan \& Joaquim Llisterri. (1999) "Diseño de corpus textuales y orales." En: Blecua, José Manuel; Gloria Claveria, Carlos Sánchez \& Joan Torruella (eds.) 1999. Filología e informática. Nuevas tecnologías en los estudios filológicos. Barcelona: Milenio, pp. 45-77.

VARGas Sierra, Chelo. (2006) "Diseño de un corpus especializado con fines terminográficos: el corpus de la piedra natural.” Debate Terminológico 2 (7/2006). Paris: RITERM (Red Iberoamericana de Terminología. Versión electrónica: $<$ http://goo.gl/bYYfE>

VARIOS AUTORES (BBC MUNDO). (2008) Glosario financiero de la crisis. Versión electrónica: <http://news.bbc.co.uk/hi/spanish/specials/2008/crisis_financiera/newsid_7330000/7330957.stm>

VARIOS AUTORES (EAGLES). (1996) "Preliminary Recommendations on Corpus Typology." Documento EAGLES (Expert Advisory Group on Language Engineering). EAGTCWG-FR2. Versión electrónica: <http://www.ilc.cnr.it/ EAGLES/corpustyp/corpustyp.html>

VARIOS AUTORES (EOSA CONSULTORES). (2007) Situación da lingua galega na sociedade: Observación no ámbito da economía e a empresa. Santiago: Observatorio da Lingua Galega. Versión electrónica: <http://www.observatoriodalinguagalega.org/files/OLG_informe_economiaempresa.pdf> 
VARIOS AUtORES (EOSA CONSUltORES). (2008a) Situación da lingua galega na sociedade: Observación no ámbito dos medios de comunicación. Santiago: Observatorio da Lingua Galega. Versión electrónica: <http://www.observatoriodalinguagalega.org/files/OLG_informe_mediosdecomunicacion.pdf>

VARIOS AUtORES (EOSA CONSUltores). (2008b) Situación da lingua galega na sociedade: Observación no ámbito da educación. Santiago: Observatorio da Lingua Galega. Versión electrónica: <http://www.observatoriodalinguagalega. org/files/OLG_informe_educacion.pdf>

VARIOS AUTORES (EXPANSIÓN). (2008) Diccionario básico de la crisis. Versión electrónica: <http://www.expansion.com/2008/03/02/inversion/1095784.html>

VARIOS AUTORES (LA CAIXA). (2011) Diccionario de términos económi$\cos$ y financieros. Versión electrónica: <https://portal.lacaixa.es/docs/ diccionario/A_es.html>

VARIOS AUTORES (RAG). (en línea) Destaques comentados: Devindicar e Devindicación. Versión electrónica: <http://academia.gal/>

ZANETTIN, Federico. (2011) "Translation and corpus design." SYNAPS, A Journal of Professional Communication 26, pp. 14-23.

ZanetTin, Federico. (2000) "Parallel Corpora in Translation Studies: Issues in Corpus Design and Analysis." En: Olohan, Maeve (ed.) 2000. Intercultural Faultines. Research Models in Translation Studies I. Textual and Cognitive Aspects. Manchester: St. Jerome, pp. 105-118.

Zanettin, Federico; Silvia Bernardini \& Dominic Stewart. (2003) Corpora in Translator Education. Manchester: St. Jerome. 


\section{NOTA BIOGRÁFICA / BIONOTE}

MARTA GARCÍA GONZÁLEZ es licenciada y doctora en Traducción e Interpretación y máster en Comercio Internacional por la Universidad de Vigo. Trabajó como traductora profesional de 1997 a 2011, principalmente en el ámbito de la traducción jurídica y económica, pero también como traductora de documentales y largometrajes. Desde 2001 es profesora del área de Traducción e Interpretación de la Facultad de Filología y Traducción de la Universidad de Vigo y Vocal de la Comisión Académica del Máster en Traducción Multimedia y del Programa de Doctorado en Comunicación de la misma universidad. En el ámbito de la investigación, es miembro del grupo GETLT y sus intereses son la traducción de textos económicos, la didáctica de la traducción y la traducción multimedia y las lenguas minorizadas.

MARTA GARCía GonZÁlez holds a degree and $\mathrm{PhD}$ in Translation and Interpreting and a MA degree in Foreign Trade by the University of Vigo. She was a professional translator from 1997 to 2010, specializing in legal and business translation. Since 2001, she has been a lecturer of Legal and Business Translation at the Faculty of Philology and Translation of the University of Vigo, where she was the Director of the faculty's MA in Multimedia Translation of that university from 2010 and 2012. Currently, she is a member of the Academic Commission of the Master in Multimedia Translation and of the $\mathrm{PhD}$ Program in Communication. She belongs to the GETLT research group of the University of Vigo and her main research interests are legal and business translation, translation pedagogy, translation from and into minoritized languages, and screen translation. 